\section{A E E T}

ASOCIACIÓN ESPAÑOLA DE ECOLOGÍA TERRESTRE

\title{
Predicciones del crecimiento en poblaciones de pino laricio (Pinus nigra Arn. ssp. salzmannii) bajo diferentes escenarios futuros de cambio climático
}

\author{
D. Candel-Pérez ${ }^{1}$, M.E. Lucas-Borja ${ }^{1}$, J.C. Linares ${ }^{2, *}$
}

(1) Departamento de Ciencia y Tecnología Agroforestal y Genética, Escuela Técnica Superior de Ingenieros Agrónomos, Universidad de Castilla-La Mancha, Campus Universitario s/n, 02071-Albacete, España

(2) Departamento de Sistemas Físicos, Químicos y Naturales, Universidad Pablo de Olavide. Ctra. Utrera km. 1, 41002-Sevilla, España.

*Autor de correspondencia: J.C. Linares [jclincal@upo.es]

> Recibido el 7 de octubre de 2012, aceptado el 31 de octubre de 2012.

\begin{abstract}
Candel-Pérez, D., Lucas-Borja, M.E., Linares, J.C. (2012). Predicciones del crecimiento en poblaciones de pino laricio (Pinus nigra Arn. ssp. salzmannii) bajo diferentes escenarios futuros de cambio climático. Ecosistemas 21(3):41-49. Doi.: 10.7818/ECOS.2012.21-3.06 La disminución de las precipitaciones y el incremento de la temperatura registrados en las últimas décadas, junto con el aumento de la probabilidad de ocurrencia de eventos extremos de sequía, hacen muy probable una mayor vulnerabilidad de las poblaciones forestales mediterráneas. Sin embargo, el impacto de los diferentes escenarios climáticos previstos para el futuro sobre el crecimiento de las especies forestales ha sido poco desarrollado. Analizamos el patrón de crecimiento del pino laricio (Pinus nigra Arn. ssp salzmannii) a lo largo de un gradiente altitudinal en la Serranía de Cuenca (este de la Península Ibérica). Estos datos, expresados como incremento de área basal, se usaron para ajustar modelos lineales mixtos de clima-crecimiento. A partir de estos modelos se realizó una predicción del crecimiento de los individuos en el futuro, usando como variables independientes los modelos de cambio climático CGCM2 y ECHAM4 y los escenarios de emisiones A2 y B2 del IPCC, disponibles a escala regional para el área de estudio. Hipotetizamos que las poblaciones situadas a menor altitud tendrán una menor tasa de crecimiento medio y serán las más vulnerables al aumento de la aridez climática. Los datos climáticos locales y regionales registrados durante el siglo XX, así como los modelos de clima futuro considerados (2011-2070), coinciden en mostrar una tendencia de calentamiento y un aumento de los episodios de sequía extrema. En las poblaciones de $P$. nigra situadas a menor altitud la tasa de crecimiento medio no fue inferior pero las correlaciones entre temperatura y crecimiento fueron siempre negativas y por tanto, los modelos predicen para estas poblaciones una drástica reducción del crecimiento y su virtual desaparición. Sin embargo, las poblaciones más envejecidas y las situadas en su límite superior altitudinal mostraron predicciones similares o ligeramente inferiores al crecimiento actual, a pesar de mostrar la menor tasa de crecimiento medio. Las muestras procedentes del centro del rango altitudinal mostraron tendencias positivas de crecimiento, ofreciendo una correlación positiva con las temperaturas de invierno y otoño. Los resultados permiten concluir que los efectos del cambio climático sobre $P$. nigra serán probablemente heterogéneos, existiendo poblaciones con alto riesgo de declive, poblaciones que aumentarían su crecimiento potencial en el futuro y poblaciones que podrían persistir de forma similar a la actualidad.
\end{abstract}

Palabras clave: CGCM2, ECHAM4, incremento de área basal, sequía, modelos climáticos, vulnerabilidad

Candel-Pérez, D., Lucas-Borja, M.E., Linares, J.C. (2012). Forecasting black pine (Pinus nigra Arn. ssp. salzmannii) growth trends under contrasting future climate change scenarios. Ecosistemas 21(3):41-49. Doi.: Doi.: 10.7818/ECOS.2012.21-3.06

Current decreasing precipitation and increasing temperatures, together with the growing likelihood of extreme drought events, may heighten the vulnerability of several tree populations in the Mediterranean. However, the effects of expected climate change on tree growth are poorly understood. We analysed growth pattern in black pine (Pinus nigra) across an altitudinal gradient in the Cuenca Mountain Range (central-eastern Spain). Data on the increment of tree basal area were used to fit climate-growth models. Then, we forecasted future tree growth using as predictors the climate data from CGCM2 and ECHAM4 models, and the IPCC emission scenarios A2 and B2, at a regional scale. We hypothesized that the populations showing the lowest mean growth rates are the most vulnerable to impending climate change. Local climatic data spanning the $20^{\text {th }}$ century, as well as the future (2011-2070) climate projections, yielded a warming trend and increasing extreme drought events. Low-elevation $P$. nigra stands did not yield the lowest growth rate but showed negative correlations among temperature and climate, and therefore, growth models forecasted growth decline and virtual extinction of these populations. Nonetheless, old-growth and higher-elevation stands yielded steady and slightly declining growth predictions, despite they showed very low mean growth rates. Core populations showed positive growth trends related to positive correlations with winter and autumn temperatures. Our results allow to expect contrasting climate change effects on $P$. nigra, where some populations are likely to decline, others populations might improve their potential growth, and some old-growth stands could endure long-term persistence in a similar manner as they currently do.

Key words: CGCM2, ECHAM4, basal-area increment, climate models, drought, vulnerability

\section{Introducción}

Las observaciones climáticas realizadas a escala global indican una tendencia general hacia el calentamiento terrestre (IPCC 2007), sin embargo existe un alto grado de incertidumbre acerca de la respuesta que ofrecerán las diferentes especies frente a los escenarios climáticos previstos para el futuro (Allen et al. 2010). Aunque algunos estudios han investigado los posibles efectos del aumento de la aridez climática en la dinámica de los bosques sensibles a la sequía (Lloret 2012; Martínez-Vilalta et al. 2012; 
Sánchez-Salguero et al. 2012a), existen pocos estudios que modelen las tendencias esperables a largo plazo en el crecimiento de especies arbóreas bajo tales condiciones (Sabaté et al. 2002; Peñuelas et al. 2010; Martín Benito et al. 2011). Los efectos climáticos en el crecimiento del árbol probablemente dependerán de los cambios específicos en las condiciones climáticas y de la sensibilidad de cada especie y su capacidad adaptativa para hacer frente a las nuevas condiciones (Felicísimo et al. 2012).

Los modelos climáticos a escala regional predicen para la cuenca mediterránea un incremento de temperatura y un aumento en los eventos extremos de precipitación (Sumner et al. 2003; IPCC, 2007). De hecho, los sistemas montañosos de la cuenca mediterránea pueden verse sometidos a incrementos de temperatura un poco más altos en comparación con las regiones circundantes (IPCC 2000, 2007), aumentando así la vulnerabilidad al cambio climático de los bosques de zonas montañosas mediterráneas (De Luis et al. 2007; Linares y Tíscar 2010, 2011; MartínBenito et al. 2010, 2011; Sánchez-Salguero et al. 2012b; Matías 2012).

Sin embargo, estas proyecciones climáticas no han sido contrastadas con las relaciones crecimiento-clima que han mostrado los árboles en las ultimas décadas, lo cual podría mejorar nuestro conocimiento sobre las tendencias esperables en el crecimiento de los individuos bajo diferente escenarios climáticos futuros. En este sentido, aunque los eventos extremos, como las sequías prolongadas, podrían tener consecuencias mucho más drásticas en el crecimiento de los árboles y su supervivencia que las que provocarían cambios graduales en las condiciones climáticas medias (Loustau et al. 2005), el modelado de las tendencias más probables puede proporcionar una valiosa información, relacionada a su vez con las tendencias en la productividad y la composición de especies (Martínez-Vilalta et al. 2008; Allen et al. 2010).

Diferentes investigadores han encontrado correlaciones tanto positivas como negativas entre el crecimiento secundario y el aumento de la temperatura (Briffa et al. 1998; Boisvenue y Running 2006; Adams et al. 2009). Estos resultados contradictorios sugieren que los escenarios climáticos previstos para el futuro podrían beneficiar a algunas poblaciones o especies forestales y a la vez inducir el declive de otras. Aunque no parece probable que el aumento de temperatura tenga un efecto positivo a escala regional en los bosques mediterráneos, donde puede ser más importante el comportamiento de las precipitaciones que el de la temperatura, resulta lógico pensar que la respuesta de las diferentes poblaciones será heterogénea (Linares y Tíscar 2011; Felicísimo 2011, 2012).

Una cuantificación de las respuestas del crecimiento radial al clima, usando datos climáticos y de crecimiento actuales y del pasado, y su proyección usando datos climáticos predichos para el futuro podría mejorar la comprensión de los mecanismos que modulan el crecimiento y la persistencia de las coníferas en zonas de montaña mediterráneas bajo diferentes escenarios de cambio climático (Linares y Tíscar 2010; Gómez-Aparicio et al. 2011; Sánchez-Salguero et al. 2012c). En este trabajo hipotetizamos que las poblaciones situadas a menor altitud tendrán una menor tasa de crecimiento medio y serán las más vulnerables al aumento de la aridez climática (ver por ejemplo Martínez-Vilalta et al. 2008). Para testar esta hipótesis se ha analizado la variabilidad del crecimiento radial y las relaciones entre clima y crecimiento de diferentes poblaciones de Pinus nigra Arn. ssp salzmannii a lo largo de un gradiente altitudinal. Nuestros objetivos específicos son: (i) modelar la relación clima-crecimiento de una especie forestal $(P$. nigra) ampliamente distribuida en la Serranía de Cuenca (centroeste de la Península Ibérica), y (ii) usar dichos modelos para predecir las tendencias en el crecimiento futuras, usando como variables independientes los modelos de cambio climático y los escenarios de emisiones disponibles a escala regional para el área de estudio.

\section{Material y Métodos}

\section{Especies y zonas de estudio}

El estudio se llevó a cabo en la Serranía de Cuenca, la cual forma parte de Sistema Ibérico Meridional, situándose entre las provincias de Cuenca y Teruel (zona centro-oriental de España; Fig.1). En esta zona la especie $P$. nigra se distribuye naturalmente entre los 1000 y 1700 m y domina la composición forestal, formando masas prácticamente puras a lo largo de este rango altitudinal, aunque también puede formar masas mixtas con otras coníferas $(P$. pinaster o $P$. halepensis en las zonas de menor altitud y $P$. sylvestris en las zonas de mayor altitud, Juniperus thurifera en zonas marginales, etc.) o frondosas (Quercus ilex, Q. faginea, etc.). En los extremos (tanto inferior como superior) del gradiente altitudinal, $P$. nigra aparece como poblaciones relictas o aisladas que están fragmentadas en pequeñas masas (Lucas-Borja et al. 2010a, 2010b). En las poblaciones de $P$. nigra estudiadas los suelos son calcáreos y generalmente poco profundos (Soil Survey Staff 1999), abarcando desde leptosoles rendzínicos a xerorthent típicos (LucasBorja et al. 2011).

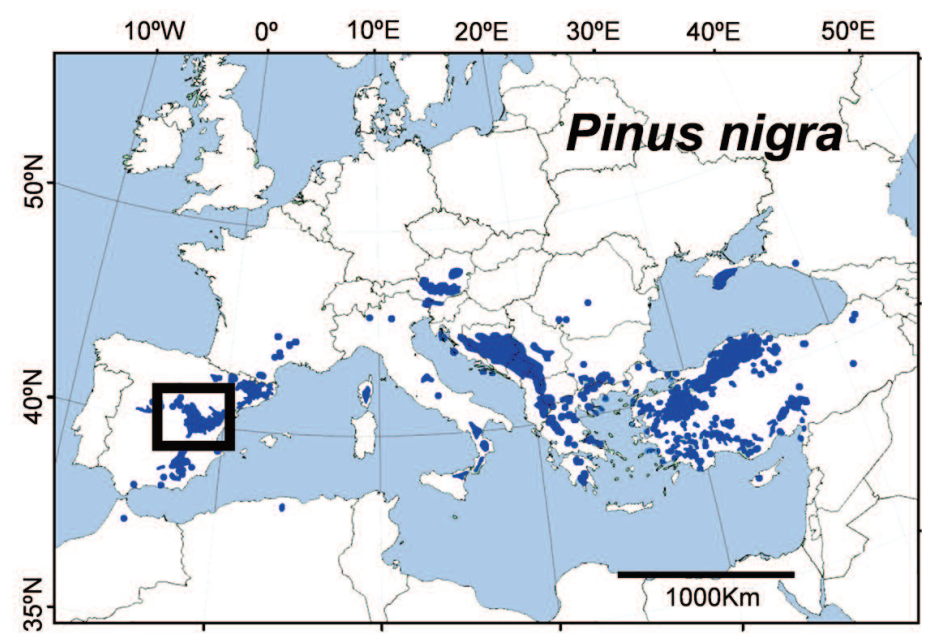

Figura 1. Distribución europea y norteafricana de la especie Pinus nigra y área de estudio en la Serranía de Cuenca (zona centro-oriental de España).

Se seleccionaron cuatro zonas de muestreo para recoger un gradiente altitudinal que reflejase el rango de distribución de $P$. nigra en la Serranía de Cuenca. Para ello se consultaron datos bibliográficos (Lucas-Borja et al. 2010b, 2011) y se realizaron varias prospecciones de campo. Las zonas finalmente seleccionadas (de menor a mayor altitud) fueron: "Arcas del Villar" (AR), "Los Palancares" (PA), "Las Majadas" (MA) y "Tragacete" (TA). Este conjunto de poblaciones se extiende desde 1100 hasta $1641 \mathrm{~m}$ (Tabla 1). Todas las masas seleccionadas proceden de regeneración natural y han sido tradicionalmente gestionadas por el método de tramos periódicos permanentes, con cortas de regeneración por aclareo sucesivo uniforme, turnos de 100 a 125 años y períodos de regeneración de 20-25 años (Fig. 2). La población situada a menor altitud (AR) presenta algunas masas mixtas de $P$. nigra con $P$. pinaster; las dos zonas situadas en la parte central del gradiente (PA y MA) constituyen masas puras de $P$. nigra; finalmente, la población situada a mayor altitud (TA) presenta poblaciones de $P$. nigra mezcladas con $P$. sylvestris.

\section{Muestreo y métodos dendrocronológicos}

En cada una de las zonas de estudio, se establecieron dos subparcelas de 0.13 ha y ubicadas a $1 \mathrm{~km}$ de distancia. Ninguna de las zonas seleccionadas mostraba evidencias de perturbaciones intensas tales como incendios forestales o tormentas, aunque en todas ellas se encontraron tocones procedentes de cortas previas. Para la cuantificación del crecimiento secundario, durante los meses de octubre y noviembre de 2010 , se extrajeron testigos del 


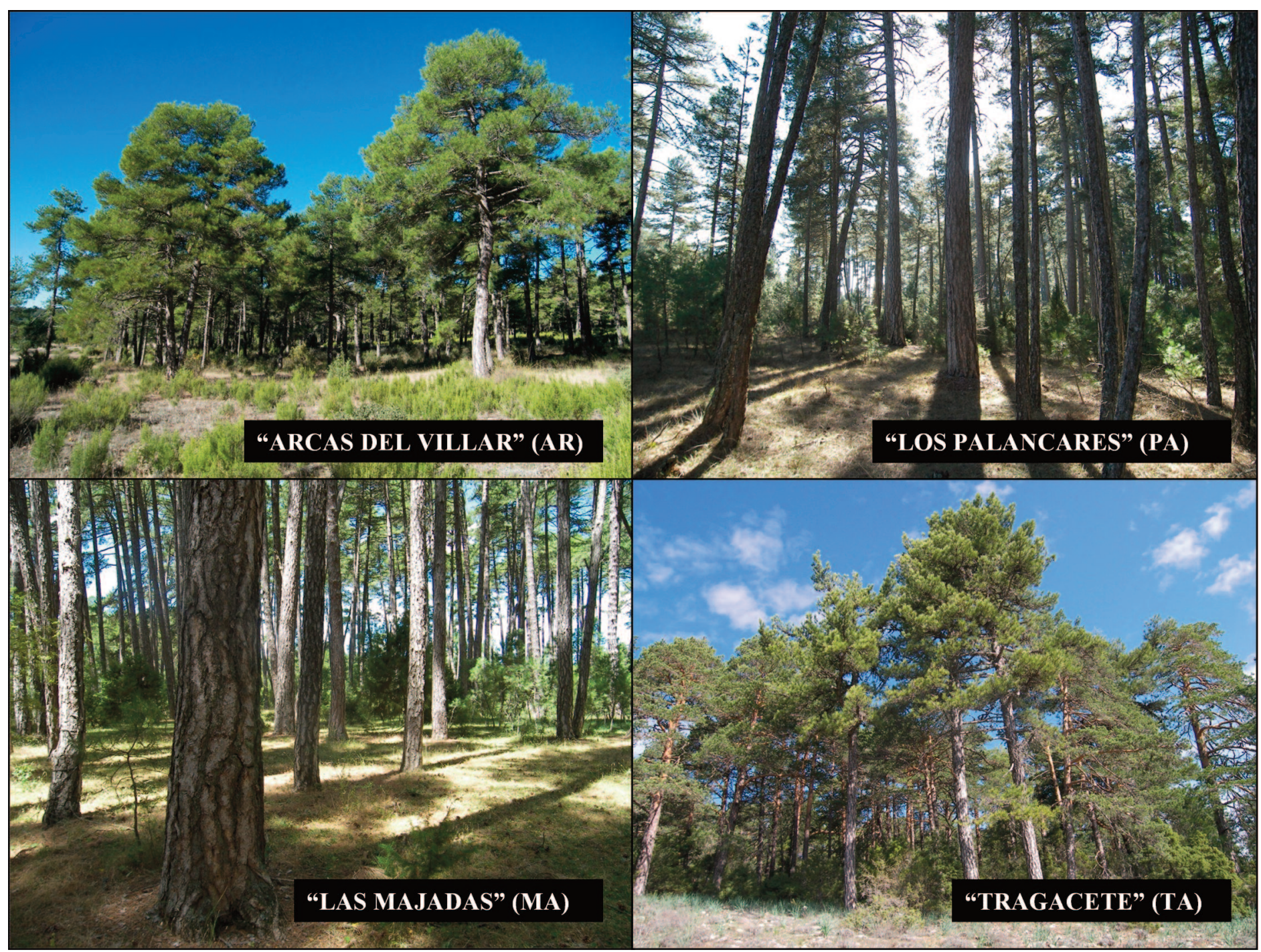

Figura 2. Imágenes de las cuatro zonas de estudio seleccionadas dentro del área de distribución de P. nigra en la Serranía de Cuenca: de menor a mayor altitud, "Arcas del Villar" (AR), "Los Palancares" (PA), "Las Majadas" (MA) y "Tragacete" (TA).

Tabla 1. Características generales de las poblaciones de Pinus nigra estudiadas en la Serranía de Cuenca (España).

\begin{tabular}{|c|c|c|c|c|}
\hline Latitud (Norte) & $39^{\circ} 54^{\prime} 24$ & $40^{\circ} 00^{\prime} 36$ & $40^{\circ} 15^{\prime} 21$ & $40^{\circ} 20^{\prime} 32$ \\
\hline Altitud (metros s.n.m.) & 1100 & 1186 & 1465 & 1641 \\
\hline Pendiente \% & 3 & 0.5 & 6.5 & 8.4 \\
\hline Temperatura media anual $1970-2011\left({ }^{\circ} \mathrm{C}\right)$ & 10.9 & 10.4 & 9.1 & 7.5 \\
\hline Precipitación total anual $1970-2011\left({ }^{\circ} \mathrm{C}\right)$ & 735 & 818 & 1004 & 1258 \\
\hline Densidad media (árboles ha-1) & 421 & 955 & 756 & 863 \\
\hline Diámetro medio a $1.3 \mathrm{~m}$ de la base de los árboles de los que se extraen tetigos $(\mathrm{cm})$ & 49 & 56 & 58 & 47 \\
\hline Edad media de los árboles de los que se extraen testigos (años a la altura del testigo) & 125 & 157 & 295 & 157 \\
\hline
\end{tabular}


tronco de 40 árboles (10 árboles dominantes en cada zona de estudio; 5 por sub-parcela). Se extrajeron dos o tres testigos por árbol a 1.3 metros de la base con barrena de Pressler, en direcciones opuestas y perpendiculares a la máxima pendiente del terreno (Fritts 1976) para evitar la presencia de madera de reacción. Se evitaron los árboles con crecimiento asimétrico y tronco no circular.

Los testigos se lijaron y se dataron visualmente mediante el reconocimiento de anillos característicos. La anchura de los anillos se midió con una precisión de $0.001 \mathrm{~mm}$ por medio de un estereomicroscopio acoplado a un dispositivo LINTAB $^{\text {TM }} 5$ RINNTECH $^{\circledR}$ Conectado a un ordenador. La calidad de la datación cruzada se comprobó utilizando el programa COFECHA (Holmes 1983). Los datos de anchura de los anillos se expresaron como incremento de área basal (IAB) por ser una variable más representativa de la cantidad de carbono destinado por el árbol al crecimiento secundario (Loustau et al. 2005).

\section{Datos climáticos}

\section{Clima reciente}

La información climática básica (valores de temperatura media mensual y precipitación total mensual) ha sido obtenida de la base de datos climáticos de alta resolución CRU (Climatic Research Unit, http://www.cru.uea.ac.uk/es) y de la Agencia Estatal de Meteorología (http://www.aemet.es/es/serviciosclimaticos/cambio climat/datos nu mericos). Hemos usado una única base de datos regional que comprende el periodo 1901-2009 (obtenido de la base de datos CRU) y al que se ha añadido, mediante correlación con los datos de la Agencia Estatal de Meteorología, los valores observados en 2010. Los datos fueron luego promediados a escala estacional (invierno: diciembre, enero y febrero; primavera: marzo, abril y mayo; verano: junio, julio y agosto; otoño: septiembre, octubre y noviembre) y normalizados (restando a cada valor la media del periodo 1960-1990 y dividiendo por la desviación estándar de ese mismo periodo) para dar a todas las variables el mismo peso relativo en los modelos de regresión, independientemente de su valor medio bruto.

\section{Modelos de clima futuro}

En este trabajo hemos usado las proyecciones climáticas realizadas con los modelos globales CGCM2 (Coupled Global Climate Model) elaborado por el Canadian Centre for Climate Modelling and Analysis y ECHAM4 (Atmospheric General Circulation Model) elaborado por el Max-Planck Institut für Meteorologie. Las proyecciones regionalizadas derivadas de estos modelos globales CGCM2 y ECHAM4 fueron realizadas por la Agencia Estatal de Meteorología (ver detalles en Brunet et al. 2009; Felicísimo 2011; Felicísimo et al. 2012). Para cada proyección o modelo se han considerado los escenarios de emisiones de gases de efecto invernadero A2 y B2 del IPCC (IPCC 2000), resultando por tanto cuatro series climáticas futuras distintas: CGCM2A2, CGCM2B2, ECHAM4A2 y ECHAM4B2. Dada la incertidumbre asociada a las proyecciones de clima futuro, hemos limitado el horizonte temporal al periodo 2011-2070 con el fin de evitar predicciones muy distantes en el tiempo, donde el efecto de factores inherentes a la biología de los individuos, tales como la edad, o la probabilidad de ocurrencia de eventos estocásticos, resulta poco predecible.

\section{Análisis estadístico}

Los datos de crecimiento (incremento de área basal) de cada individuo y la serie climática regional (datos estacionales de temperatura y precipitación normalizados) para el periodo 1960-2010 fueron utilizados para construir un modelo de crecimiento-clima en Pinus nigra. El rango temporal se limitó a periodo posterior a 1960 para asegurar que todos los individuos muestreados han superado la fase juvenil de crecimiento. Se utilizaron modelos lineales mixtos para analizar el efecto de las variables climáticas sobre el crecimiento, incluyendo las variables climáticas como factores fijos y cada individuo como un factor aleatorio (sobre el que se realizan medidas repetidas del crecimiento a lo largo del tiempo); el efecto del diámetro del árbol fue testado también, incluyéndolo como fac- tor aleatorio en lugar de la referencia del árbol. La selección de variables climáticas incluidas en el modelo y de la estructura del factor aleatorio (árbol ó diámetro) se realizó de forma manual mediante una regresión por pasos hacia atrás (incluyendo todas las variables y reduciendo progresivamente las menos significativas) hasta minimizar el valor de AIC (criterio de información de Akaike; Burnham y Anderson 2002).

La estructura de auto-correlación temporal de primer orden de los datos fue modelada a partir de los residuos no climáticos (diferencia entre los valores de IAB medidos en cada individuo para cada año y los valores de IAB predichos por el modelo para ese individuo ese mismo año). Para ello se correlacionaron los residuos no climáticos del individuo j en el año i $\left(I A B_{j i}\right)$ y los valores de crecimiento del año previo $I A B_{j(i-1)}$. De esta forma, parte de la varianza no explicada por las variables climáticas queda recogida por la estructura de auto-correlación temporal de los datos y ésta es también aplicada a los datos de crecimiento futuro, puesto que el crecimiento predicho por el modelo en el año previo es conocida, por ejemplo, el valor de crecimiento observado en 2010 supone el primer valor de crecimiento previo introducido en la predicción futura de 2011; el valor predicho (ya no observado) para 2011 es introducido como valor previo para generar la predicción del año 2012, y así sucesivamente.

El mismo procedimiento se llevó a cabo con la edad de los individuos, de modo que en caso de obtenerse un efecto significativo de la edad sobre el crecimiento, una vez que el efecto del clima ha sido eliminado (modelado), éste puede incluirse y proyectarse igualmente a los datos de crecimiento futuro, puesto que la edad de los individuos en cada año es fácilmente estimada sumando uno al valor de edad del árbol en el año previo, por ejemplo, el valor de edad (medido mediante dendrocronología) en la fecha de muestreo (2010) más uno es el valor de edad del individuo en la predicción de 2011; el valor de edad en 2011 más uno será introducido como edad en la predicción del año 2012, y así sucesivamente.

\section{Resultados}

Los datos climáticos regionales muestran un incremento de la temperatura de invierno de aproximadamente $1.4^{\circ} \mathrm{C}$ para el periodo 1901-2000; los modelos climáticos considerados predicen un incremento de entre $2.3^{\circ} \mathrm{C}(\mathrm{ECHAM} 4 \mathrm{~A} 2)$ y $1.0^{\circ} \mathrm{C}$ (CGCM2B2) para el periodo 2010-2070, pasando la temperatura media de invierno de $4.9^{\circ} \mathrm{C}$ para el periodo $1901-2000$ a un valor de entre $6.5^{\circ} \mathrm{C}$ y $7^{\circ} \mathrm{C}$ para el periodo $2010-2070$ (Fig. 3a). La temperatura de primavera no mostró una tendencia significativa durante el periodo 1901-2000, sin embargo, los modelos climáticos considerados predicen un incremento de entre $2.4^{\circ} \mathrm{C}$ (ECHAM4A2) y $1.0^{\circ} \mathrm{C}$ (CGCM2B2) para el periodo 2010-2070, pasando la temperatura media de primavera de $11.2^{\circ} \mathrm{C}$ para el periodo $1901-2000$ a un valor de entre $13.6^{\circ} \mathrm{C}$ y $11.9^{\circ} \mathrm{C}$ en el periodo $2010-2070$. La temperatura de verano tampoco muestra una tendencia significativa durante el periodo 1901-2000; en este caso los modelos climáticos considerados predicen un incremento de entre $3.9^{\circ} \mathrm{C}$ (CGCM2A2) y $2.4^{\circ} \mathrm{C}$ (CGCM2B2) para el periodo 2010-2070, pasando la temperatura media de verano de $18.7^{\circ} \mathrm{C}$ para el periodo $1901-2000$ a un valor de entre $21.8^{\circ} \mathrm{C}$ y 21.3 ${ }^{\circ} \mathrm{C}$ en el periodo $2010-2070$. La temperatura de otoño se incrementó aproximadamente $1.5^{\circ} \mathrm{C}$ durante el periodo 1901-2000, mientras que los modelos climáticos predicen un incremento de entre $3.4^{\circ} \mathrm{C}$ (ECHAM4A2) y $1.3^{\circ} \mathrm{C}$ (CGCM2B2) para el periodo 2010-2070, pasando la temperatura media de otoño de $9^{\circ} \mathrm{C}$ en el siglo $\mathrm{XX}$ a un valor de entre $10.8^{\circ} \mathrm{C}-11.6^{\circ} \mathrm{C}$ en el periodo $2010-2070$. Las tendencias arriba descritas suponen en promedio un aumento de la temperatura media anual de entre $2.9^{\circ} \mathrm{C}$ (ECHAM4A2) y $1.4{ }^{\circ} \mathrm{C}$ (CGCM2B2) para el periodo 2010-2070, pasando la temperatura media anual de $11.1^{\circ} \mathrm{C}$ para el periodo $1901-2000$ a un valor de entre $12.7^{\circ} \mathrm{C}$ y $13.3^{\circ} \mathrm{C}$ en el periodo $2010-2070$ (Fig. 3a)

En el caso de la precipitación, los datos climáticos regionales muestran un ligero descenso, de aproximadamente $55 \mathrm{~mm}$ en la precipitación total anual para el periodo 1901-2000. Sin embargo los modelos climáticos considerados muestran una notable varia- 
a)

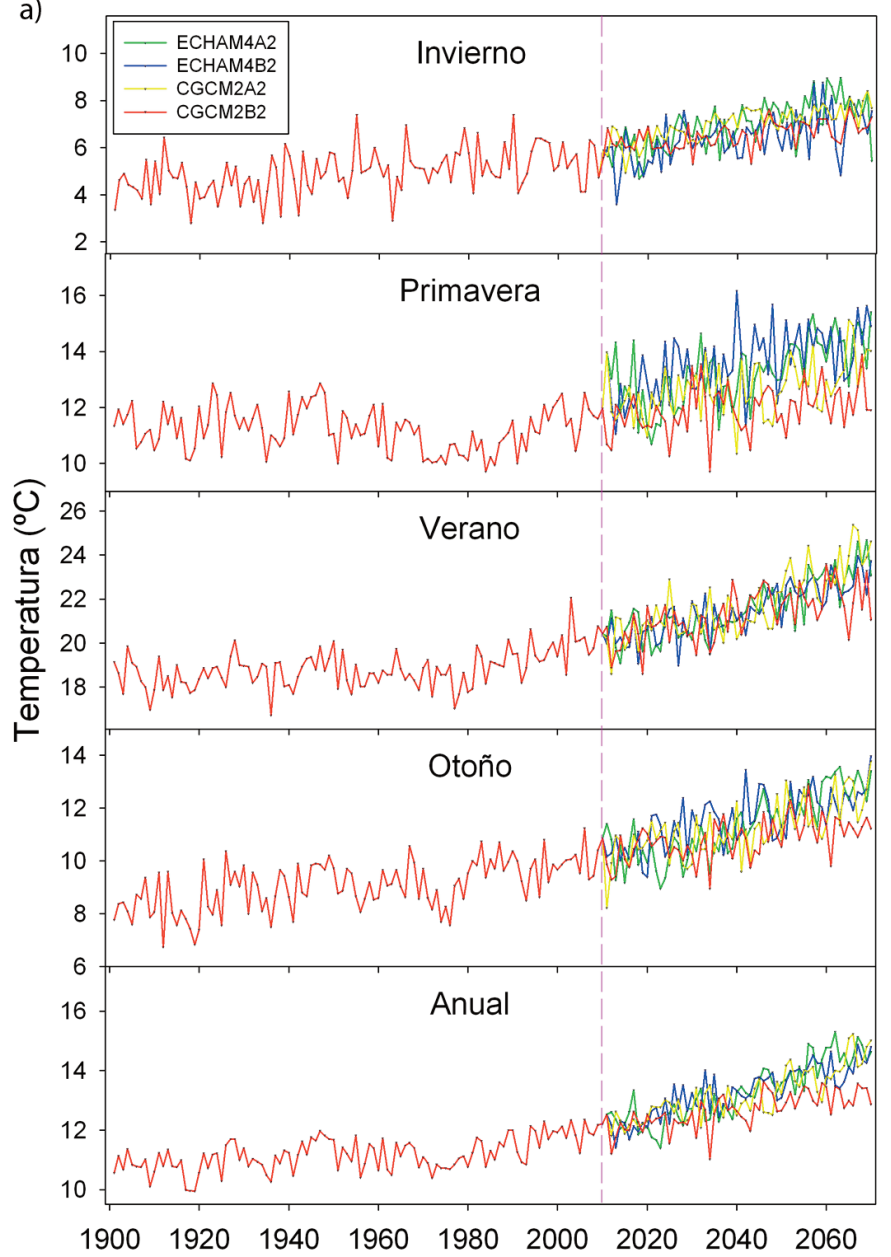

b)

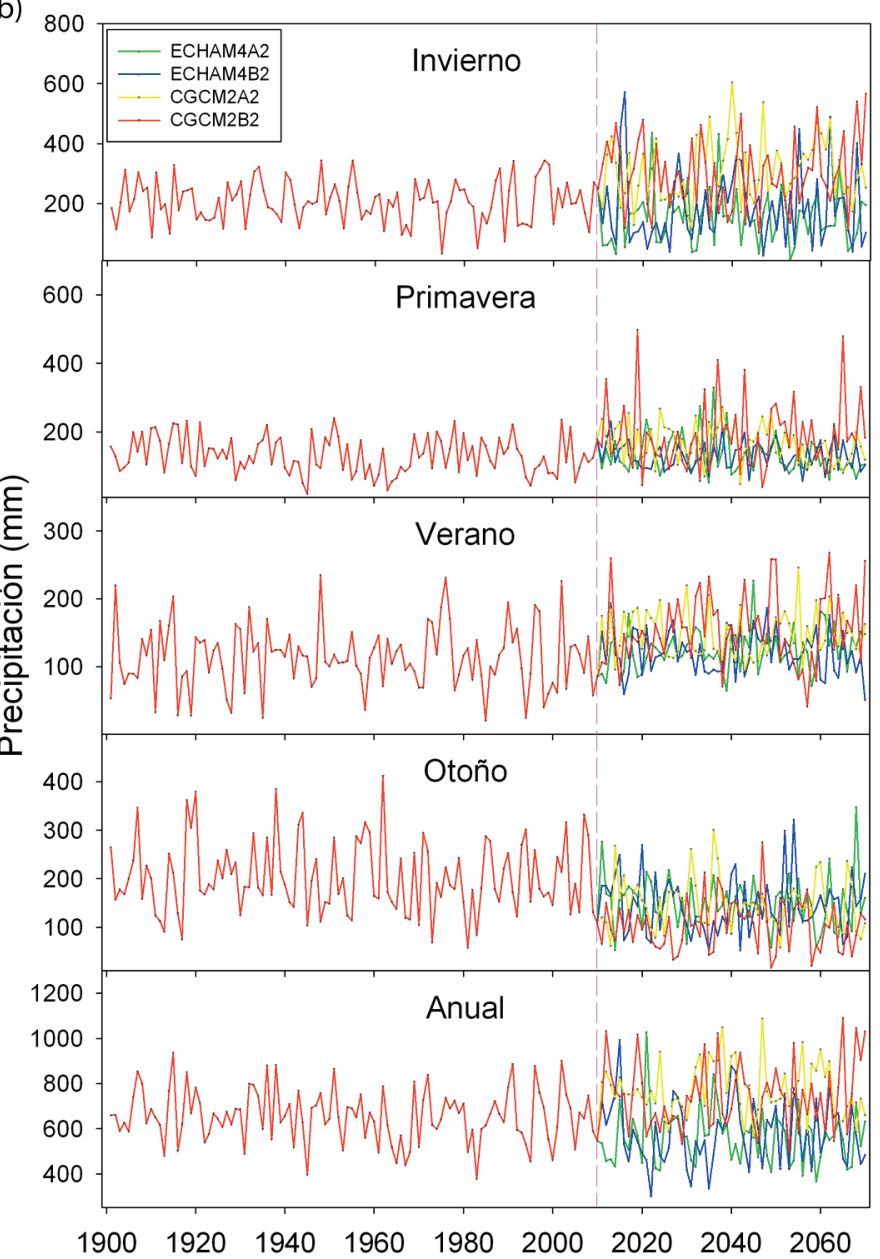

Figura 3. Valores regionales de temperatura media (a) y precipitación total (b) registrados en el área de estudio para el periodo 1901-2010 y predichos por los modelos climáticos para el periodo 2011-2070; la línea vertical indica la transición entre valores observados y predichos.

bilidad en las predicciones. En general, los modelos ECHAM4 predicen descensos en las precipitaciones (entre $20 \mathrm{~mm}$ y $30 \mathrm{~mm}$ para la precipitación total anual durante el periodo 2010-2070), especialmente notables en primavera (Fig. 3b). Por el contrario, los modelos del grupo CGCM2 predicen tanto tendencias positivas (por ejemplo en la precipitación de primavera en el modelo CGCM2B2) como negativas (precipitación de primavera en el modelo CGCM2A2). En general todos los modelos predicen un aumento el la frecuencia y la magnitud de los eventos extremos (Fig. 3b). La precipitación total anual podría oscilar entre 570 (ECHAM4) y los $750 \mathrm{~mm}$ (CGCM2) en el periodo 2010-2070, mientras que la media en el siglo XX fue de unos $650 \mathrm{~mm}$ (Fig. 3b).

Los modelos de crecimiento-clima explicaron entre un $18.5 \%$ y un $65 \%$ de varianza, mientras que la estructura de auto-correlación temporal de los datos explicó entre un $3.9 \%$ y un $13.3 \%$ (Tabla 2); la edad no fue significativa en ninguna población. Los individuos situados en los extremos del gradiente altitudinal (AR a menor altitud, TA en el límite altitudinal superior) mostraron correlaciones negativas con la temperatura del otoño previo al año de crecimiento. La temperatura de invierno mostró una correlación negativa con el crecimiento en los individuos situados a menor altitud, mientras que dicha correlación fue positiva en las poblaciones PA y TA. La temperatura de verano fue significativa en todas las poblaciones, mostrando en todos los casos una correlación negativa con el crecimiento, mayor a menor altitud (Tabla 2). La precipitación de otoño mostró una correlación positiva con el crecimiento en las poblaciones AR y MA; mientras que en la población PA este efecto se obtuvo para la precipitación de invierno y verano; la población situada a mayor altitud (TA) sólo mostró correlaciones significativas con la temperatura. En conjunto (sumando los efectos de las variables climáticas y la estructura de auto-correlación) los modelos de crecimiento explicaron
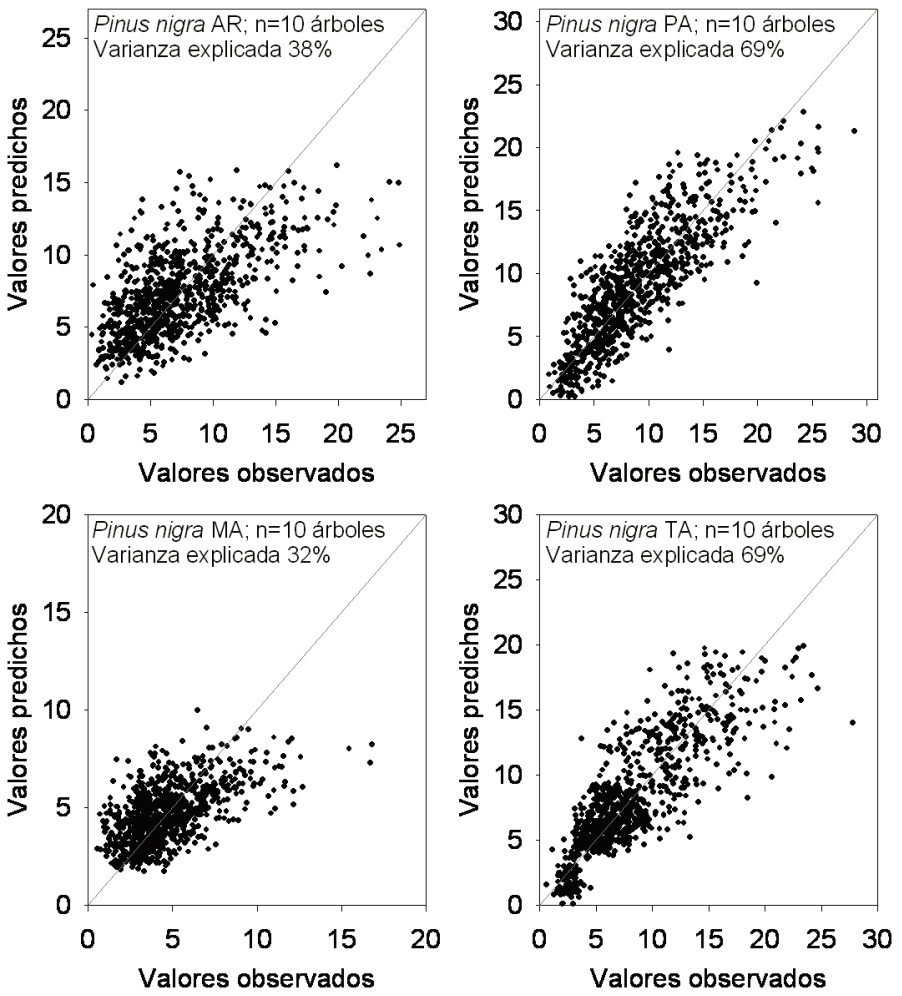

Figura 4. Correlación entre los valores observados y predichos por los modelos de crecimiento sumando los efectos de las variables climáticas y la estructura de auto-correlación. Para cada parcela se indica el porcentaje de varianza explicado por el modelo (ver también la Tabla 2). 
Tabla 2. Estadísticos de las variables climáticas incluidas en los modelos lineales mixtos utilizados para ajustar y predecir el crecimiento secundario en cuatro poblaciones de Pinus nigra (AR, PA, MA, y TA, ver también la Tabla 1). La auto-correlación de primer orden ha sido incluida en el modelo usando como factor el crecimiento (incremento de área basal) en el año previo, ajustado sobre los residuos no climáticos. El mismo procedimiento se usa para incluir en el modelo el efecto de la edad del árbol; ns indica que la variable no es estadísticamente significativa.

\begin{tabular}{|c|c|c|c|c|c|}
\hline Población & $\begin{array}{l}\text { Variables climáticas } \\
\text { incluidas en el modelo }\end{array}$ & $\begin{array}{l}\text { Valor del coeficiente } \\
\text { de regresión }\end{array}$ & Error estándar & $\begin{array}{l}\text { Peso relativo de la } \\
\text { variable en el modelo (\%) }\end{array}$ & $\begin{array}{c}\text { Varianza explicada } \\
(\%)\end{array}$ \\
\hline \multirow{4}{*}{ AR } & T otoño previo & -0.55 & 0.52 & 13.23 & 5.07 \\
\hline & T verano & -1.83 & 0.24 & 44.13 & 16.91 \\
\hline & P otoño & 0.73 & 0.21 & 17.68 & 6.78 \\
\hline & Auto-correlación primer orden & 0.25 & 0.02 & 17.02 & 6.52 \\
\hline \multirow{5}{*}{ PA } & T invierno & 1.36 & 0.14 & 15.29 & 10.59 \\
\hline & T primavera & -1.13 & 0.37 & 12.67 & 8.77 \\
\hline & T verano & -1.18 & 0.24 & 13.30 & 9.21 \\
\hline & T otoño & 2.62 & 0.51 & 29.43 & 20.38 \\
\hline & $P$ invierno & 0.80 & 0.17 & 9.03 & 6.25 \\
\hline \multirow{5}{*}{ MA } & T verano & -0.62 & 0.16 & 17.72 & 5.63 \\
\hline & T otoño & 0.95 & 0.36 & 27.14 & 8.63 \\
\hline & P otoño & 0.47 & 0.13 & 13.30 & 4.23 \\
\hline & Auto-correlación primer orden & 0.35 & 0.02 & 41.85 & 13.31 \\
\hline & Edad & ns & ns & ns & ns \\
\hline Total & & & & 100 & 31.80 \\
\hline \multirow{5}{*}{ TA } & T otoño previo & -2.67 & 0.41 & 64.33 & 44.41 \\
\hline & T invierno & 0.67 & 0.13 & 16.05 & 11.08 \\
\hline & T verano & -0.58 & 0.19 & 13.96 & 9.64 \\
\hline & Auto-correlación primer orden & 0.17 & 0.01 & 5.66 & 3.91 \\
\hline & Edad & ns & ns & ns & ns \\
\hline
\end{tabular}
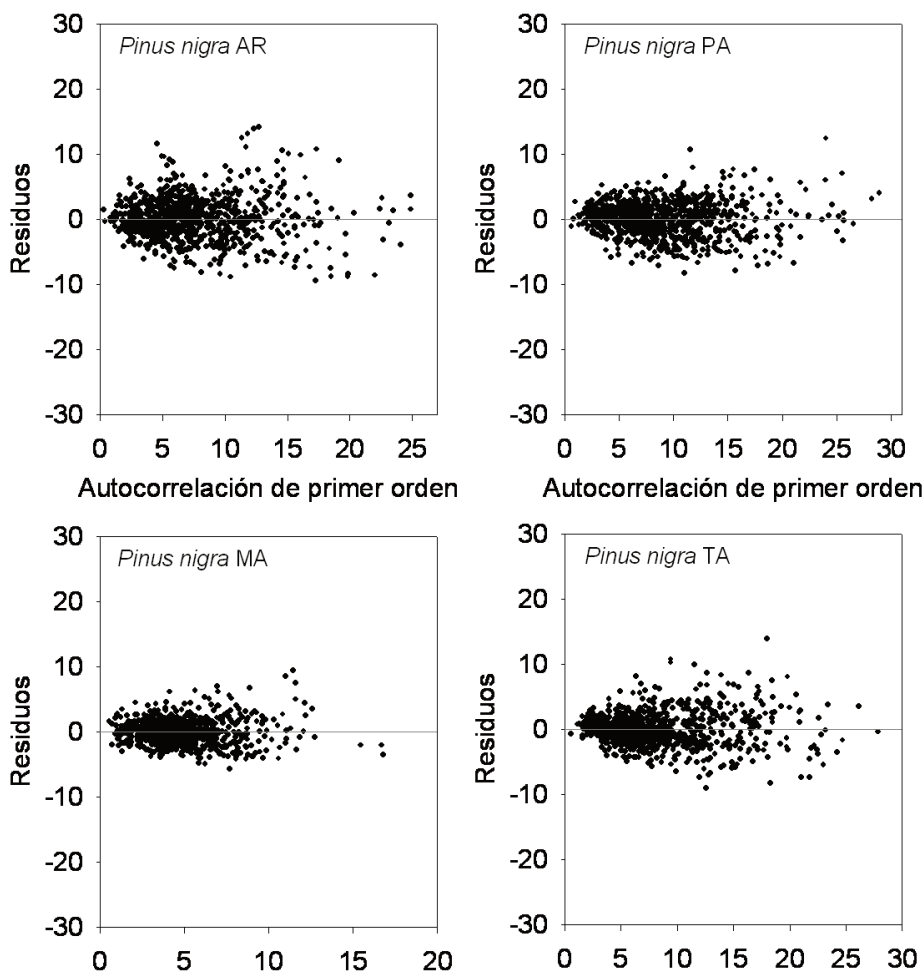

Autocorrelación de primer orden

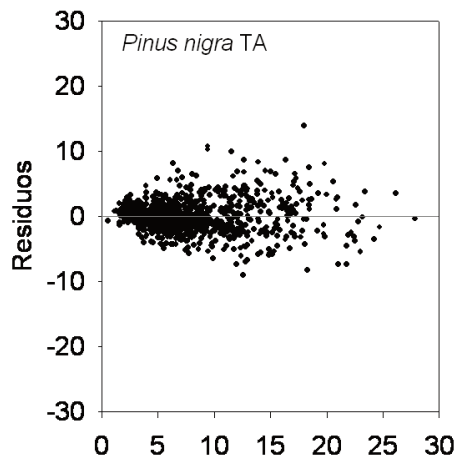

Autocorrelación de primer orden
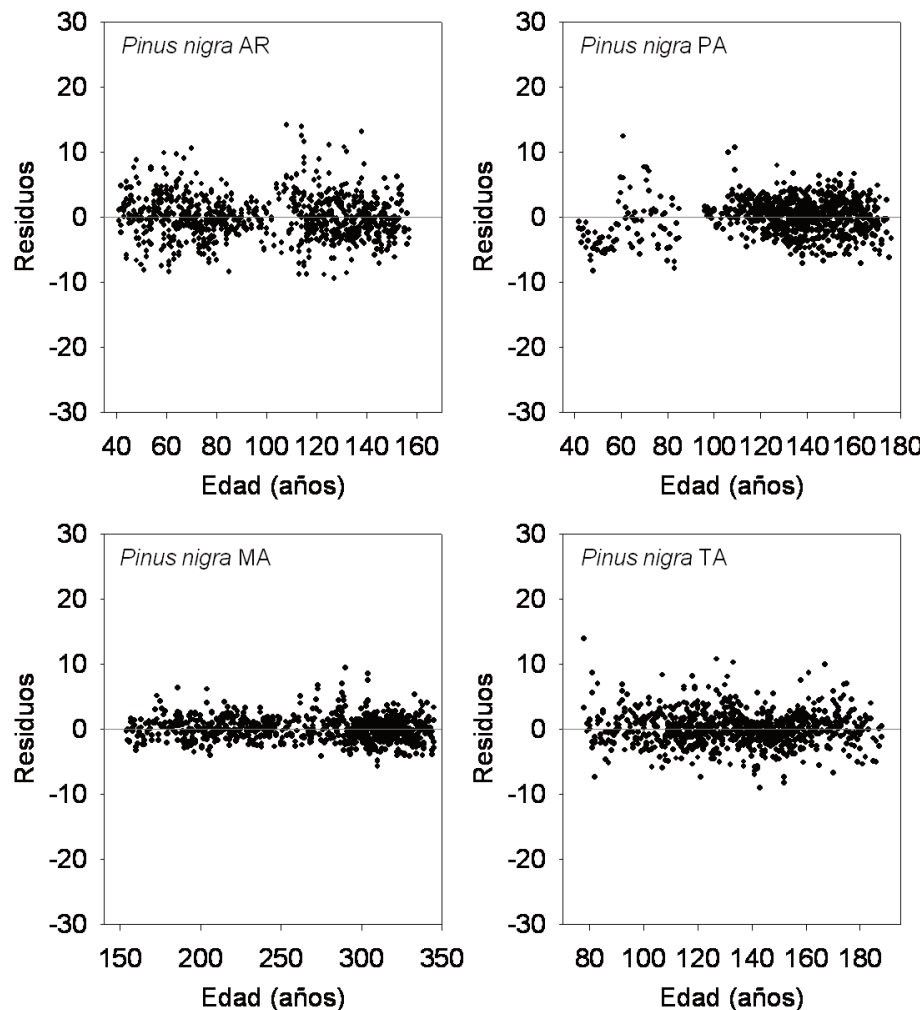

Figura 5. Correlación entre los valores de crecimiento del año previo, auto-correlación de primer orden; (a), la edad de los individuos (b) y los residuos (diferencia entre los valores observados y predichos) de los modelos de crecimiento sumando los efectos de las variables climáticas y la estructura de auto-correlación. 


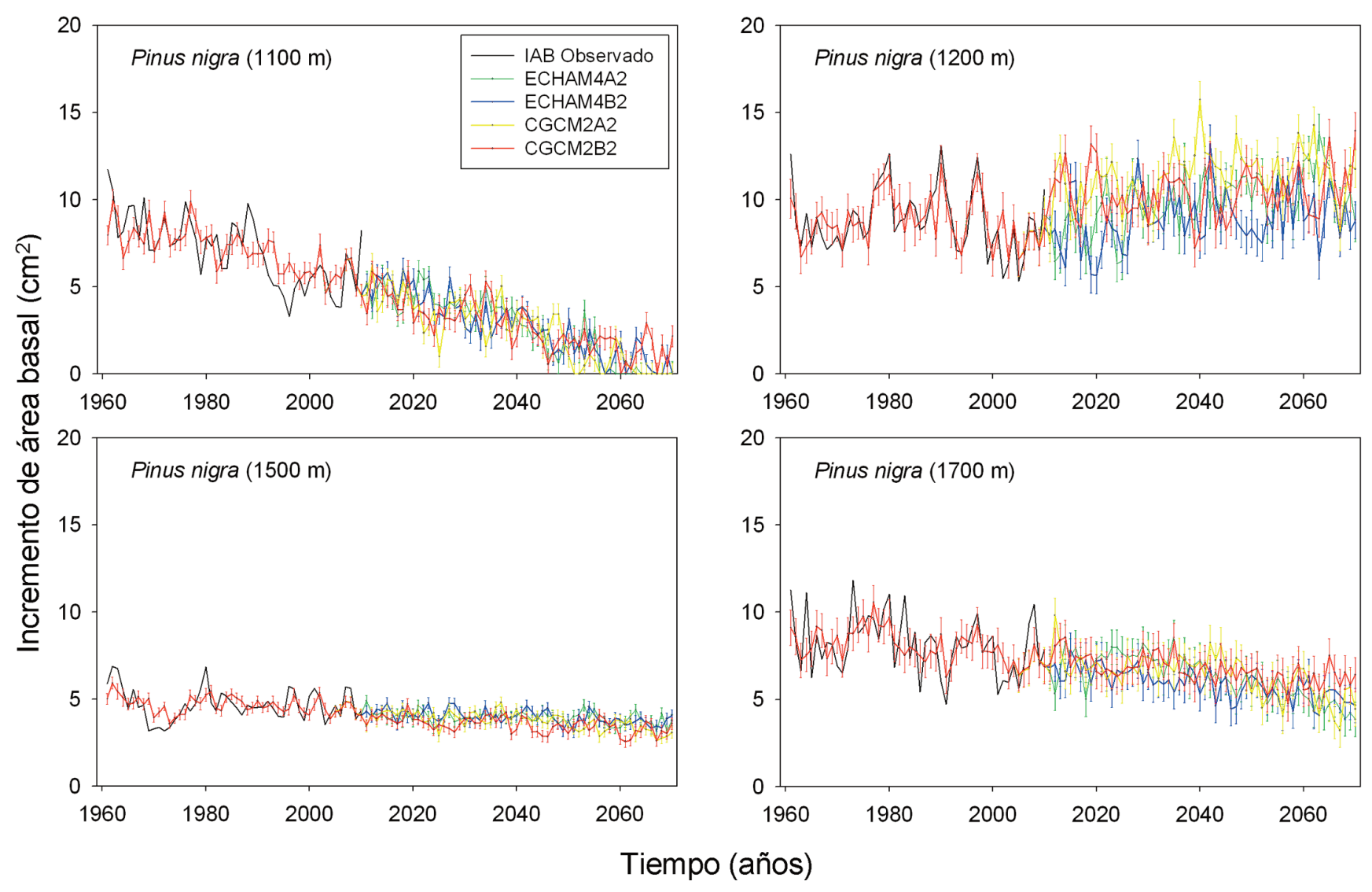

Figura 6. Valores de crecimiento (incremento de área basal, IAB) observados (línea negra) y predichos por los diferentes modelos climáticos: ECHAM4A2, línea verde; ECHAM4B2, línea azul; CGCM2A2, línea amarilla; CGCM2B2, línea roja. Las barras de error indican el error estándar.

entre un $32 \%$ y un $69 \%$ de varianza (Fig. 4). El análisis de los residuos de los modelos de crecimiento muestra que tanto el efecto de auto-correlación (Fig. 5a) como el efecto de la edad (Fig. 5b) no están correlacionados con la varianza no explicada.

En las poblaciones de $P$. nigra situadas a menor altitud $(A R$, 1100m, Fig. 6), los modelos de crecimiento predicen una drástica reducción del crecimiento, llegando incluso a ser cero hacia el año 2050 y, por tanto, esperándose su virtual desaparición. Sin embargo, en el resto de poblaciones, las tendencias de crecimiento predichas son similares a las actuales o ligeramente superiores en el caso de PA. La población formada por individuos más viejos (MA, ver la edad media de los individuos en la Tabla 1), y la situada en el límite superior de distribución (TA), muestran los valores más bajos de crecimiento, sin embargo no presentan una mayor vulnerabilidad, comparadas con la situada a menor altitud, cuya tasa de crecimiento en la segunda mitad del siglo XX era significativamente superior (Fig. 6).

\section{Discusión}

Nuestros resultados confirman una tendencia de calentamiento durante el siglo XX en la zona de estudio, así como una mayor probabilidad de períodos de sequía extrema hacia el final del siglo XX. Estas tendencias aparecen reforzadas en los modelos de cambio climático considerados en este trabajo para el periodo 2011-2070 (Figs. 3a y $3 b$ ). Asimismo, se muestra como las relaciones entre clima y crecimiento de Pinus nigra son heterogéneas a lo largo de su rango altitudinal de distribución (Tabla 2; véase también GómezAparicio et al. 2011; Sánchez-Salguero et al. 2012c; Linares y Tíscar 2010, 2011; Martínez-Vilalta et al. 2008; Lebourgeois 2000; Fernández et al. 1996). El aumento de las temperaturas de invierno podría ampliar el período de crecimiento disponible (Wullschleger et al. 2002; Boisvenue y Running 2006), sin embargo, una disminución simultánea en la disponibilidad hídrica puede reducir el crecimiento total, debido a la disminución en la tasa de fijación de carbono que induce la sequía (McDowell et al. 2010).
De forma general, se podrían esperar un mayor crecimiento en aquellos sitios actualmente limitados por bajas temperaturas (es decir, las poblaciones situadas a mayor altitud) como resultado del calentamiento (Tardif et al. 2003; Büntgen et al. 2007). Sin embargo, nuestros resultados no confirman esta hipótesis, ya que los individuos situados a mayor altitud muestran una tendencia de crecimiento descendente, como consecuencia de su correlación negativa con las temperaturas del otoño previo y de verano (Tabla 2). También hay que señalar que los individuos muestreados en TA presentan exposición sur (S y SE), por lo que son zonas comparativamente más cálidas y más secas que las de laderas con exposición norte.

Los individuos muestreados en el límite inferior de distribución (AR, Tabla 1) mostraron correlaciones negativas entre crecimiento y temperatura, por ejemplo, con el otoño del año previo. Menor IAB después de un otoño cálido sugieren una disminución en la acumulación de carbohidratos (o un aumento del gasto respiratorio) y la disminución del tamaño de la zona del cambium, desarrollado durante el otoño anterior (Richter et al. 1991; Fernández et al. 1996). En contraste con los individuos de $P$. nigra situados a menor altitud, los individuos muestreados en la parte central del gradiente (PA) muestran una correlación positiva con las temperaturas de invierno y de otoño.

El aumento de la temperatura de verano incrementa el estrés hídrico y disminuye el crecimiento de $P$. nigra a lo largo de todo su rango de distribución. Esta respuesta también se ha observado en otras especies. Por ejemplo, reducciones del crecimiento radial en respuesta a la sequía han sido identificadas por Eilmann et al. (2006) en Pinus sylvestris. Por otra parte, aunque el crecimiento secundario de $P$. sylvestris ha aumentado durante el siglo $\mathrm{XX}$ en Cataluña (NE de España), el incremento de las temperaturas también ha afectado negativamente a los sitios más secos (MartínezVilalta et al. 2008, 2012; Fernández-Cancio et al. 2011; Gómez-Aparicio et al. 2011; Lloret 2012; Sánchez-Salguero et al. 2012b, 2012c). El calentamiento climático y el estrés hídrico severo 
parecen ser las principales causas en la disminución del crecimiento en los bosques de Abies alba cerca del límite meridional de la distribución de la especie (Camarero et al. 2011; Macías et al. 2006) y también parece estar relacionado con una reducción del crecimiento en poblaciones de Abies pinsapo, $P$. nigra y Cedrus atlantica del sur de España y el norte de Marruecos, según diversos estudios dendrocronológicos (Linares et al. 2010; Allen et al. 2010; Martín-Benito et al. 2011; Linares y Tíscar, 2011).

Puesto que nuestro área de estudio ha sido históricamente objeto de una gestión forestal bajo diferentes intensidades de manejo, por medio de métodos tales como los aclareos sucesivos uniformes, es previsible que exista una interacción entre la estructura de la masa forestal, las relaciones clima-crecimiento que hemos modelado y la evolución que previsiblemente tendrán estas poblaciones en el futuro (Camarero et al. 2011; Gómez-Aparicio et al. 2011; Lines et al. 2010; Martín-Benito et al. 2010, 2011). En este sentido, las masas situadas a mayor altitud (MA y TA) presentaron los individuos más viejos (Tabla 1). La edad de los individuos podría tener un papel fundamental en las diferencias de varianza explicada por el clima, los valores medios de IAB y las tendencias en el crecimiento (Linares y Tíscar 2010; Tíscar 2002, 2007). Los valores medios de IAB son menores en los árboles más viejos (MA), y la varianza total explicada por el clima menor. Estos resultados podrían deberse a cambios endógenos, relacionados con la edad, y que afectan a las relaciones hídricas y el balance de carbono del árbol (Hunt et al. 1999). Tales cambios ontogenéticos afectan a la xilogénesis (Rossi et al. 2008) y alteran las relaciones clima-crecimiento (Carrer y Urbinati 2004). El aumento de tamaño y la complejidad estructural de los árboles más viejos elevan los gastos de respiración de mantenimiento y disminuyen la eficiencia hídrica, lo que puede explicar una menor tasa de crecimiento y bajas correlaciones clima-crecimiento (véase también Ryan y Yoder 1997).

\section{Agradecimientos}

Queremos mostrar nuestro agradecimiento a L. Cayuela y a dos revisores anónimos, cuyos comentarios mejoraron una versión previa del manuscrito. Este trabajo ha sido financiado por la Junta de Comunidades de Castilla-La Mancha [POII10-0112-7316]. La Consejería de Agricultura de ésta comunidad proporcionó los medios necesarios para la realización de los trabajos de campo. D. Candel agradece al Fondo Social Europeo y la Junta de Comunidades de Castilla-La Mancha la concesión de una beca FPI.

\section{Referencias}

Adams, H.D., Guardiola-Claramonte, M., Barron-Gafford, G.A., Villegas, J.C., Breshears, D.D., Zou, C.B., Troch, P.A., Huxman, T.E., 2009. Temperature sensitivity of drought-induced tree mortality: implications for regional die-off under global-change-type drought. Proceedings of the National Academy of Sciences USA 106:7063-7066.

Allen, C.D., Macalady, A.K., Chenchouni, H., Bachelet, D., McDowell, N., Vennetier, M., Kitzberger, T., Rigling, A., Breshears, D.D., Hogg, E.H., Gonzalez, P., Fensham, R., Zhang, Z., Castro, J., Demidova, N., Lim, J.H., Allard, G., Running, S.W., Semerci, A., Cobb, N., 2010. A global overview of drought and heat-induced tree mortality reveals emerging climate change risks for forests. Forest Ecology and Management 259:660-684.

Boisvenue, C., Running, S.W., 2006. Impacts of climate change on natural forest productivity-evidence since the middle of the 20th century. Global Change Biology 12:1-12.

Briffa, K.R., Schweingruber, F.H., Jones, P.D., Osborn, T.J., Shiyatov, S.G., Vaganov, E.A., 1998. Reduced sensitivity of recent tree-growth to temperature at high northern latitudes. Nature 391:678-682.

Brunet, M., Casado, M.J., De Castro, M., Galán, P., López, J.A., Martín, J.M., Pastor, A., Petisco, E., Ramos, P., Ribalaygua, J., Rodríguez, E., Sanz, I., Torres, L., 2009. Generación de escenarios regionalizados de cambio climático para España. AEMET. Madrid, España..

Büntgen, U., Frank, D.C., Kaczka, R.J., Verstege, A., Zwijackz-Kozica, T., Esper, J., 2007. Growth responses to climate in a multi-species tree- ring network in the Western Carpathian Tatra Mountains, Poland and Slovakia. Tree Physiolog. 27:689-702.

Burnham, KP., Anderson, DR. 2002. Model Selection and Multimodel Inference: a Practical Information-Theoretic Approach. Springer, Heidelberg, Germany.

Camarero, J.J., Bigler, C.J., Linares, J.C., Gil-Pelegrín, E., 2011. Synergistic effects of past historical logging and drought on the decline of Pyrenean silver fir forests. Forest Ecology and Management. 262:759-769.

Carrer, M., Urbinati, C., 2004. Age-dependent tree ring growth responses to climate of Larix decidua and Pinus cembra in the Italian Alps. Ecology 85:730-740.

De Luis, M., Gričar, J., Čufar, K., Raventós, J., 2007. Seasonal dynamics of wood formation in Pinus halepensis from dry and semi-arid ecosystems in Spain. IAWA Journal. 28:389-404.

Eilmann, B., Weber, P., Rigling, D., Eckstein, D., 2006. Growth reactions of Pinus sylvestris $\mathrm{L}$. and Quercus pubescens Willd. to drought years at a xeric site in Valais, Switzerland. Dendrochronologia 23:121-132.

Felicísimo, A.M. (coord.), 2011. Impactos, vulnerabilidad y adaptación al cambio climático de la biodiversidad española. 1. Flora y vegetación. Oficina Española de Cambio Climático, Ministerio de Medio Ambiente y Medio Rural y Marino. Madrid, España.

Felicísimo, A.M., Muñoz, J., Mateo, R.G. 2012. Vulnerabilidad de la flora y vegetación españolas ante el cambio climático y propuestas de actuación. Ecosistemas 21(3):1-6.

Fernández-Cancio, A., Navarro Cerrillo, R.M., Sánchez Salguero, R., Fernández Fernández, R., Manrique Menéndez, E., 2011. Viabilidad fitoclimática de las repoblaciones de pino silvestre (Pinus sylvestris L.) en la Sierra de los Filabres (Almeria). Ecosistemas 20(1):124-144.

Fernández, A., Génova, M., Creus, J., Gutiérrez, E., 1996. Dendroclimatological investigation covering the last 300 years in central Spain, En: Dean, J.S., Meko, D.M., Swetman, T.W. (Eds.), Tree rings, environment and humanity, pp. 181-190, Radiocarbon. University of Arizona, Tucson, USA.

Fritts, H.C., 1976. Tree rings and Climate. Blackburn Press, Caldwell, New Jersey, USA.

Gómez-Aparicio, L., García-Valdes, R., Ruiz-Benito, P., Zavala, M.A., 2011. Disentangling the relative importance of climate, size and competition on tree growth in Iberian forests: implications for management under global change. Global Change Biology 17:2400-2414.

Holmes, R.L., 1983. Computer-assisted quality control in tree-ring dating and measurement. Tree-Ring Bulletin 43:68-78.

Hunt, E.R., Lavigne, M.B., Franklin, S.E., 1999. Factors controlling the decline of net primary production with stand age for balsam fir in Newfoundland assessed using an ecosystem simulation model. Ecological Modelling 122:151-164.

IPCC, 2000. Informe especial del IPCC. Escenarios de emisiones. Grupo Intergubernamental de expertos sobre cambio climático. Cambridge University Press, London, UK.

IPCC, 2007. Climate change, fourth assessment report. Cambridge University Press, London, UK.

Lebourgeois, F., 2000. Climatic signals in earlywood, latewood and total ring width of Corsican pine from western France. Annals of Forest Science 57:55-164.

Linares, J.C., Tíscar, P.A., 2010. Climate change impacts and vulnerability of the southern populations of Pinus nigra subsp. salzmannii. Tree Physiology 30:795-806.

Linares, JC., Tíscar, PA., 2011. Buffered climate change effects in a Mediterranean pine species: range limit implications from a tree-ring study. Oecologia 167:847-859.

Linares, J.C., Camarero, J.J., Carreira, J.A., 2010. Competition modulates the adaptation capacity of forests to climatic stress: Insights from recent growth decline and death in relict stands of the Mediterranean fir Abies pinsapo. Journal of Ecology 98:592-603.

Lines, ER., Coomes, DA., Purves, DW., 2010. Influences of Forest Structure, Climate and Species Composition on Tree Mortality across the Eastern US. PLOS ONE. 5:e13212.

Lloret, F., 2012. Vulnerabilidad y resiliencia de los ecosistemas forestales frente a episodios extremos de sequía. Ecosistemas 21(3):85-90.

Loustau, D., Bosc, A., Colin, A., Ogée, J., Davi, H., François, C., Dufrêne, E., Déqué, M., Cloppet, E., Arrouays, D., Le Bas, C., Saby, N., Pignard, G., Hamza, N., Granier, A., Bréda, N., Ciais, P., Viovy, N., Delage, F., 2005. Modelling climate change effects on the potential production of French plains forests at the sub-regional level. Tree Physiology 25:813-823. 
Lucas-Borja, M.E., Bastida, F., Nicolás, C., Moreno, J.L., Del Cerro, A., Andrés, M., 2010a. Influence of forest cover and herbaceous vegetation on the microbiological and biochemical properties of soil under Mediterranean humid climate. European Journal of Soil Biology 46:273-279.

Lucas-Borja, M.E., Silva-Santos, P., Fonseca, T.F., Tíscar Oliver, P.A., López-Serrano, F.R., Andrés Abellán, M., Martínez García, E., Del Cerro Barja, A. 2010b. Modelling Spanish black pine postdispersal seed predation in Central-Eastern Spain. Forest Systems 19(3):393-403.

Lucas-Borja, M.E., Fonseca, T., Parresol, B.R., Silva-Santos, P., García-Morote, F.A., Tíscar-Oliver, P.A., 2011. Modelling Spanish black pine seedling emergence: Establishing management strategies for endangered forest areas. Forest Ecology and Management 262:195-202.

Macías, M., Andreu, L., Bosch, O., Camarero, J.J., Gutiérrez, E., 2006. Increasing aridity is enhancing silver fir Abies alba (Mill.) water stress in its south-western distribution limit. Climatic Change 79:289-313.

Martín-Benito, D., Heinrich, I., Helle, G., del Río, M., Cañellas I., 2010. Response of climate-growth relationships and water use efficieny to thinning in a Pinus nigra afforestation. Forest Ecology and Management 259:967-975.

Martín-Benito, D., Kint, V., del Río, M., Muys, B., Cañellas I., 2011. Growth responses of West- Mediterranean Pinus nigra to climate change are modulated by competition and productivity: past trends and future perspetives. Forest Ecology and Management 262:1030-1040.

Martínez-Vilalta, J., López, B.C., Adell, N., Badiella, L., Ninyerola, M., 2008. Twentieth century increase of Scots pine radial growth in NE Spain shows strong climate interactions. Global Change Biology 14:2868-2881.

Martínez-Vilalta, J., Aguadé, D., Banqué, M., Barba, J., Curiel Yuste, J., Galiano, L., Garcia, N., Gómez, M.,Hereş, A.M., López, B.C., Lloret, F., Poyatos, R., Retana, J., Sus, J., Vayreda, J., Vilà-Cabrera, A., 2012. Las poblaciones ibéricas de pino albar ante el cambio climático: con la muerte en los talones. Ecosistemas 21(3):15-21.

Matías, L. 2012. Cambios en los límites de distribución de especies arbóreas como consecuencia de las variaciones climáticas. Ecosistemas 21(3):91-96.

McDowell, N., Allen, C.D., Marshall, L., 2010. Growth, carbon isotope discrimination, and climate-induced mortality across a Pinus ponderosa elevation transect. Global Change Biology 16:399-415.

Peñuelas, J., Gracia, C., Filella, I., Jump, A., Carnicer, J., Coll, M., Lloret, F., Yuste, J.C., Estiarte, M., Rutishauser, T., Ogaya, R., Llusià, J., Sardans, J., 2010. Introducing the climate change effects on Mediterranean forest ecosystems: observation, experimentation, simulation, and management. Forêt méditerranéenne XXXI 357-362.
Richter, K., Eckstein, D., Holmes, R.L., 1991. The dendrochronological signal of pine trees (Pinus spp.) in Spain. Tree-Ring Bulletin 51:1-13.

Rossi, S., Deslauriers, A., Anfodillo, T., Carrer, M., 2008. Age-dependent xylogenesis in timberline conifers. New Phytologist 177:199-208.

Ryan, M.G., Yoder, B.J., 1997. Hydraulic limits to tree height and tree growth. BioScienc. 47:235-242.

Sabaté, S., Gracia, C., Sánchez, A., 2002. Likely effects of climate change on growth of Quercus ilex, Pinus halepensis, Pinus pinaster, Pinus sylvestris and Fagus sylvatica forests in the Mediterranean Region. Forest Ecology and Management 162:23-37.

Sánchez-Salguero, R., Navarro-Cerrillo, R.M., Camarero, J.J., FernándezCancio, A., Swetnam, T.W., Zavala, M.A., 2012a. Vulnerabilidad frente a la sequía de repoblaciones de dos especies de pinos en su límite meridional en Europa. Ecosistemas 21(3):31-40.

Sánchez-Salguero, R., Navarro-Cerrillo, R.M., Swetnam, T.W., Zavala, M.A., $2012 \mathrm{~b}$. Is drought the main decline factor at the rear edge of Europe? The case of southern Iberian pine plantations. Forest Ecology and Management 271:158-169.

Sánchez-Salguero, R., Navarro-Cerrillo R.M., Camarero J.J., FernándezCancio A., 2012c. Selective drought-induced decline of pine species in southeastern Spain. Climatic Change 113:767-785.

Soil Survey Staff, 1999. Soil taxonomy: a basic system of soil classification for making and interpreting soil surveys $\left(2^{\text {ed }}\right)$. United States Department of Agriculture, Washington, USA.

Sumner, G., Romero, R., Homar, V., Ramis, C., Alonso, S., Zorita, E., 2003. An estimate of the effects of climate change on the rainfall of Mediterranean Spain by the late twenty first century. Climate Dynamics 20:789805.

Tardif, J., Camarero, J.J., Ribas, M., Gutiérrez, E., 2003. Spatiotemporal variability in tree growth in the Central Pyrenees: climatic and site influences. Ecological Monographs 73:241-257.

Tíscar, P., 2002. Capacidad reproductiva de Pinus nigra subsp. salzmannii en relación con la edad de la planta madre. Investigación Agraria: Sistemas y Recursos Forestales 11(2):357-371.

Tíscar, P., 2007. Dinámica de regeneración de Pinus nigra subsp. salzmannii al sur de su área de distribución: etapas, procesos y factores implicados. Investigación Agraria: Sistemas y Recursos Forestales 16(2):124-135.

Wullschleger, S.D., Tschaplinski, T.J., Norby, R.J., 2002. Plant water relations at elevated $\mathrm{CO}_{2}$ - implications for water-limited environments. Plant, Cell and Environment 25:319-331. 